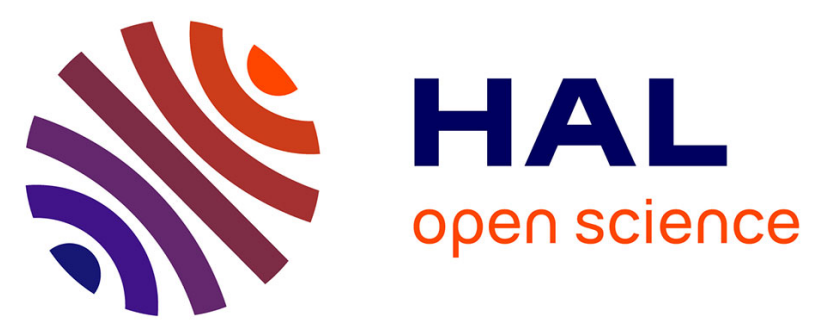

\title{
Reaching the quantum limit of sensitivity in electron spin resonance
}

A Bienfait, Y Kubo, M Stern, Xin Zhou, T Schenkel, D Vion, D Esteve, B Julsgaard, K Mølmer, P. Bertet, et al.

\section{- To cite this version:}

A Bienfait, Y Kubo, M Stern, Xin Zhou, T Schenkel, et al.. Reaching the quantum limit of sensitivity in electron spin resonance. Nature Nanotechnology, 2015, 11, pp.253-257. 10.1038/NNANO.2015.282 . cea-01366689

\section{HAL Id: cea-01366689 https://hal-cea.archives-ouvertes.fr/cea-01366689}

Submitted on 15 Sep 2016

HAL is a multi-disciplinary open access archive for the deposit and dissemination of scientific research documents, whether they are published or not. The documents may come from teaching and research institutions in France or abroad, or from public or private research centers.
L'archive ouverte pluridisciplinaire HAL, est destinée au dépôt et à la diffusion de documents scientifiques de niveau recherche, publiés ou non, émanant des établissements d'enseignement et de recherche français ou étrangers, des laboratoires publics ou privés. 


\title{
Reaching the quantum limit of sensitivity in electron spin resonance
}

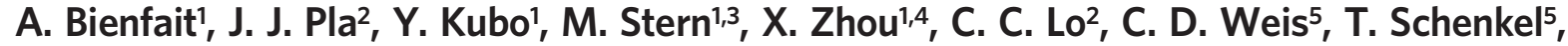 \\ M. L. W. Thewalt ${ }^{6}$, D. Vion', D. Esteve', B. Julsgaard7, K. Mølmer', J. J. L. Morton² and P. Bertet ${ }^{1 \star}$
}

The detection and characterization of paramagnetic species by electron spin resonance (ESR) spectroscopy is widely used throughout chemistry, biology and materials science ${ }^{1}$, from in vivo imaging ${ }^{2}$ to distance measurements in spin-labelled proteins $^{3}$. ESR relies on the inductive detection of microwave signals emitted by the spins into a coupled microwave resonator during their Larmor precession. However, such signals can be very small, prohibiting the application of ESR at the nanoscale (for example, at the single-cell level or on individual nanoparticles). Here, using a Josephson parametric microwave amplifier combined with high-quality-factor superconducting microresonators cooled at millikelvin temperatures, we improve the state-of-the-art sensitivity of inductive ESR detection by nearly four orders of magnitude ${ }^{4,5}$. We demonstrate the detection of 1,700 bismuth donor spins in silicon within a single Hahn ${ }^{6}$ echo with unit signal-to-noise ratio, reduced to 150 spins by averaging a single Carr-PurcellMeiboom-Gill sequence ${ }^{7}$. This unprecedented sensitivity reaches the limit set by quantum fluctuations of the electromagnetic field instead of thermal or technical noise, which constitutes a novel regime for magnetic resonance. The detection volume of our resonator is $\sim 0.02 \mathrm{nl}$, and our approach can be readily scaled down further to improve sensitivity, providing a new versatile toolbox for ESR at the nanoscale.

A wide variety of techniques are being actively explored to push the limits of the sensitivity of electron spin resonance (ESR) to the nanoscale, including approaches based on optical ${ }^{8,9}$ or electrical ${ }^{10,11}$ detection, as well as scanning probe methods ${ }^{12,13}$. Our focus in this work is to maximize the sensitivity of inductively detected pulsed ESR to maintain the broad applicability to different spin species as well as fast high-bandwidth detection. Pulsed ESR spectroscopy proceeds by probing a sample coupled to a microwave resonator of frequency $\omega_{0}$ and quality factor $Q$ with sequences of microwave pulses that perform successive spin rotations, triggering the emission of a microwave signal called a spin-echo whose amplitude and shape contain the desired information about the number and properties of paramagnetic species. The spectrometer sensitivity is conveniently quantified by the minimal number of spins $N_{\min }$ that can be detected within a single echo ${ }^{6}$. Conventional ESR spectrometers use threedimensional resonators with moderate quality factors in which the spins are only weakly coupled to the microwave photons and thus obtain a sensitivity of $N_{\min } \approx 1 \times 10^{13}$ spins at $T=300 \mathrm{~K}$ and $\mathrm{X}$-band frequencies $\left(\omega_{0} / 2 \pi \approx 9-10 \mathrm{GHz}\right)$. To increase the sensitivity, micro-fabricated metallic planar resonators with smaller mode volumes have been used, resulting in larger spin-microwave coupling ${ }^{14,15}$. Combined with operation at $T=4 \mathrm{~K}$ and the use of low-noise cryogenic amplifiers and superconducting high- $Q$ thinfilm resonators, sensitivities up to $N_{\min } \approx 1 \times 10^{7}$ spins have been reported, which represents the current state of the art ${ }^{4,5,16}$.

Further improvements in the sensitivity of ESR spectroscopy can be obtained by cooling the sample and resonator down to millikelvin temperatures that satisfy $T \ll \hbar \omega_{0} / k_{\mathrm{B}}$ at X-band frequencies. As a result, both the spins and the microwave field reach their quantum ground state, which is the optimal situation for magnetic resonance because the spins are then fully polarized and thermal noise is suppressed. The noise in the emitted echo signal is essentially due to vacuum quantum fluctuations of the microwave field, with a dimensionless spectral power density of $n_{\mathrm{eq}}=S(\omega) /(\hbar \omega)=1 / 2$, possibly supplemented by extra noise $n_{\mathrm{s}}$ due to the spontaneous emission of the spins (Supplementary Section IV). However, the total noise spectral density in the detected signal $n=n_{\mathrm{eq}}+n_{\mathrm{s}}+n_{\mathrm{amp}}$ also includes the added noise $n_{\mathrm{amp}}$ of the first amplifier of the detection chain. Benefiting from the low noise afforded by low-temperature operation thus requires nearly noiseless amplifiers at microwave frequencies, as were recently developed in the context of superconducting quantum circuits. These Josephson parametric amplifiers (JPAs) are operated at millikelvin temperatures, have a bandwidth of up to $\sim 100 \mathrm{MHz}$, and a low saturation input power (typically $1-10 \mathrm{fW})^{17,18}$. They have been shown to add the minimum amount of noise permitted by quantum mechanics ${ }^{19}: n_{\mathrm{amp}}=0.5$ when both field quadratures are equally amplified (non-degenerate mode $)^{17}$ and $n_{\mathrm{amp}}=0$ when only one quadrature is amplified (degenerate mode $)^{20}$. JPAs have been used so far for reading out the state of superconducting qubits ${ }^{21}$, the motion of nanomechanical oscillators ${ }^{22}$ and the charge state of a quantum $\operatorname{dot}^{23}$, as well as for high-sensitivity magnetometry ${ }^{24}$. Here, we show that they are also well suited to amplifying the weak and narrowband signals emitted by small numbers of spins, with the ultimate sensitivity allowed by quantum mechanics, enabling us to demonstrate a four orders of magnitude improvement in sensitivity over the state of the art.

We use an ensemble of Bi donors implanted over a $150 \mathrm{~nm}$ depth into an isotopically enriched ${ }^{28} \mathrm{Si}$ crystal, on top of which is patterned a superconducting $\mathrm{Al}$ thin-film microresonator consisting of an interdigitated capacitor in parallel with a wire inductance (see Fig. 1 for a sketch of the set-up). Due to this geometry, the microwave field $B_{1} \cos \omega_{0} t$ couples only to the $N_{\mathrm{Bi}} \simeq 4 \times 10^{7}$ implanted $\mathrm{Bi}$ atoms located in the area below the wire. The

\footnotetext{
'Quantronics Group, SPEC, CEA, CNRS, Université Paris-Saclay, CEA Saclay, 91191 Gif-sur-Yvette, France. ${ }^{2}$ London Centre for Nanotechnology, University College London, London WC1H OAH, UK. ${ }^{3}$ Quantum Nanoelectronics Laboratory, BINA, Bar Ilan University, Ramat Gan, Israel. ${ }^{4}$ ISEN Department, Institute of Electronics Microelectronics and Nanotechnology, CNRS UMR 8520, Avenue Poincaré, CS 60069, Villeneuve d'Ascq Cedex 59652, France. ${ }^{5}$ Accelerator Technology and Applied Physics Division, Lawrence Berkeley National Laboratory, Berkeley, California 94720, USA. ${ }^{6}$ Department of Physics, Simon Fraser University, Burnaby, British Columbia V5A 156, Canada. ${ }^{7}$ Department of Physics and Astronomy, Aarhus University, Ny Munkegade 120, Aarhus C DK-8000, Denmark. *e-mail: patrice.bertet@cea.fr
} 


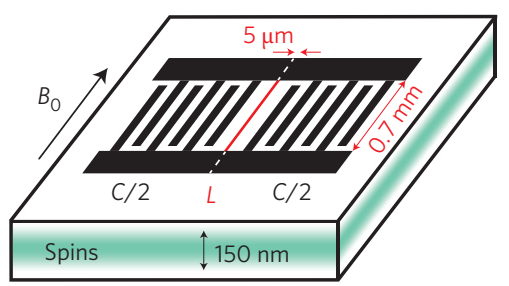

b

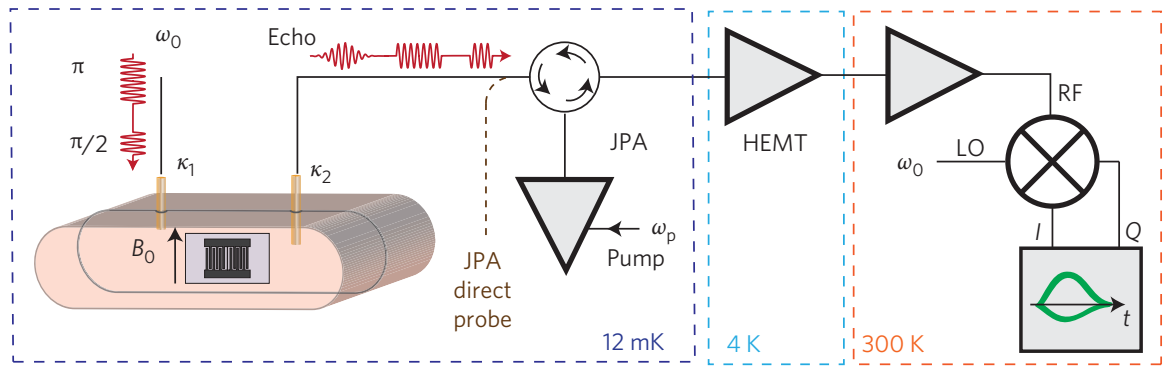

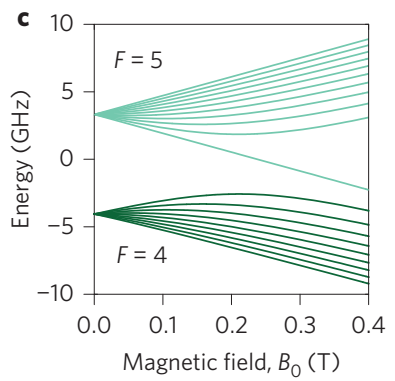

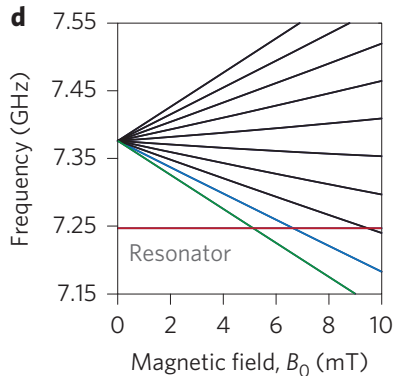

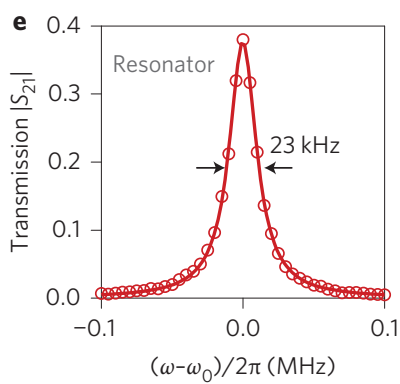

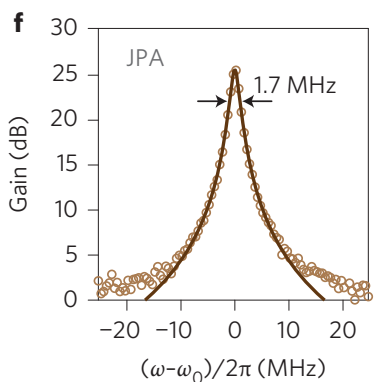

Figure 1 | Experimental set-up and spin system. a, The Al microwave resonator with frequency $\omega_{0}$ consists of an interdigitated capacitor in parallel with a 5 - $\mu \mathrm{m}$-wide wire inductor, fabricated on a Bi-doped ${ }^{28} \mathrm{Si}$ epi-layer. $\mathbf{b}$, The sample is mounted in a Cu box, thermally anchored at $12 \mathrm{mK}$, and probed by microwave pulses via asymmetric antennas coupled to the resonator with rates $\kappa_{1}=1.2 \times 10^{4} \mathrm{~s}^{-1}$ and $\kappa_{2}=5.6 \times 10^{4} \mathrm{~s}^{-1}$. A magnetic field $B_{0}$ is applied parallel to the resonator inductance. Microwave pulses at $\omega_{0}$ are sent by antenna 1 , and the microwave signal leaving via antenna 2 is directed to the input of a JPA. The JPA is powered by a pump signal at $\omega_{\mathrm{p}} \approx 2 \omega_{0}$, and its output is further amplified at $4 \mathrm{~K}$ by a HEMT amplifier, followed by amplification and demodulation at room temperature, yielding the two field quadratures $I(t), Q(t)$. c, Energy levels of Bi donors in Si, expressed in units of frequency (see spin Hamiltonian in Supplementary Section II). d, ESR-allowed transitions in the low-field limit. For $B_{0} \leq 8 \mathrm{mT}$, the $\left|F, m_{\mathrm{F}}\right\rangle=|4,-4\rangle \rightarrow|5,-5\rangle$ and $|4,-3\rangle \rightarrow|5,-4\rangle$ transitions cross the resonator frequency at $B_{0}=5$ and $7 \mathrm{mT}$, respectively. e, Measured resonator transmission coefficient $\left|S_{21}\right|$ (red circles), yielding $\omega_{0} / 2 \pi=7.24 \mathrm{GHz}$ and a total quality factor $Q=3 \times 10^{5}$ (red curve is a fit). $\mathbf{f}$, The JPA can be characterized via a direct line bypassing the resonator, yielding a gain, in non-degenerate mode, of $\mathrm{G}>20 \mathrm{~dB}$ above a $3 \mathrm{MHz}$ bandwidth. Circles are experimental data and curve is a Lorentzian fit.

sample is inserted inside a $\mathrm{Cu}$ box to suppress the resonator radiative losses while enabling its transmission to be probed by capacitive coupling to input and output antennas. In this well-controlled environment the resonator reaches a loaded quality factor of $Q=3 \times 10^{5}$ for frequency $\omega_{0} / 2 \pi=7.24 \mathrm{GHz}$ (Fig. 1e). Microwave pulses at $\omega_{0}$ are applied to the cavity input, and the output signal (including the echoes emitted by the spins) is directed towards the input of a JPA operated in reflection ${ }^{18}$ with power gain $G$ up to $\sim 23 \mathrm{~dB}$ at $\omega_{0}$ when powered by a pump microwave signal at frequency $\omega_{\mathrm{p}} \approx 2 \omega_{0}, \omega_{\mathrm{p}}=2 \omega_{0}$ corresponding to the degenerate mode of operation $^{18}$. The signal reflected and amplified from the JPA is then further amplified by a semiconducting high-electron-mobility transistor (HEMT) amplifier at $4 \mathrm{~K}$, and finally demodulated at frequency $\omega_{0}$, yielding time traces for the two quadratures of $I(t)$, $Q(t)$ (see Supplementary Section I for more details). The sample and JPA are cooled at $12 \mathrm{mK}$ in a dilution refrigerator.

An in-plane magnetic field $B_{0}$ is applied parallel to the sample surface along the resonator inductance. Neutral $\mathrm{Bi}$ donors in $\mathrm{Si}$ have an $S=1 / 2$ electron spin coupled by a strong hyperfine interaction term $A \mathbf{S} \cdot \mathbf{I}$ to the $I=9 / 2$ nuclear spin of ${ }^{209} \mathrm{Bi}$ (refs 25,26), with $A / h=1.48 \mathrm{GHz}$. In the low-field regime, the 20 electro-nuclear energy states are best described by their total angular momentum $\mathbf{F}=\mathbf{S}+\mathbf{I}$ and its projection $m_{\mathrm{F}}$; they can be grouped in an $F=4$ ground and an $F=5$ excited multiplet separated by a frequency of $5 \mathrm{~A} / \mathrm{h}=7.38 \mathrm{GHz}$ in zero field (Fig. 1). With the chosen orientation of $B_{0}$, the $B_{1}$ microwave field generated by the resonator is perpendicular to the spin quantization axis and only transitions obeying $\left|\Delta m_{\mathrm{F}}\right|=1$ have a significant matrix element (Fig. 1d) for $B_{0} \leq 10 \mathrm{mT}$. Their frequency in the $\sim 7.3-7.5 \mathrm{GHz}$ range makes $\mathrm{Bi}: \mathrm{Si}$ an ideal system for coupling to superconducting $\mathrm{Al}$ resonators, which can only withstand fields below $\sim 10 \mathrm{mT}$.
In our case, the $\left|F, m_{\mathrm{F}}\right\rangle=|4,-4\rangle \rightarrow|5,-5\rangle$ and $|4,-3\rangle \rightarrow|5,-4\rangle$ transitions are expected to be resonant with $\omega_{0}$ at $B_{0}=5$ and $7 \mathrm{mT}$, respectively; corresponding peaks in the integrated spin-echo signal (of duration $T_{\mathrm{E}} \approx 20 \mu \mathrm{s}$ ) are indeed measured as shown in Fig. 2a-c. Each transition consists of two sub-peaks, with an inhomogeneous linewidth $\Gamma / 2 \pi=2 \mathrm{MHz}$. We attribute this substructure to the differential strain ${ }^{27}$ acting on the $\mathrm{Bi}$ atoms lying just under the wire versus those around it (Supplementary Section III). We will focus in the following on the $|4,-4\rangle \rightarrow|5,-5\rangle$ transition for the spins lying under the wire, at $B_{0}=5.18 \mathrm{mT}$. Well-defined Rabi oscillations are observed in the integrated echo signal as a function of the refocusing pulse amplitude (Fig. 2b), with a $100 \mathrm{kHz}$ Rabi frequency for a remarkably low input power of $3 \mathrm{pW}$ (ref. 4). The decay of the integrated echo signal as a function of the total delay $2 \tau$ between the initial $\pi / 2$ pulse and the echo is well fitted by an exponential decay with a time constant $T_{2}=10 \mathrm{~ms}$, a typical coherence time for $\mathrm{Bi}^{28} \mathrm{Si}$ (ref. 28; Fig. 2d). Given the high quality factor of the resonator, the $\pi / 2$ pulse has an exponential tail with a characteristic time $\tau=2 / \kappa=14 \mu$ s, so the minimum $T_{2}$ that one could measure in this set-up is $\sim 50 \mu \mathrm{s}$. The energy relaxation time $T_{1}$ is measured by the inversion recovery method to be $T_{1}=0.3 \mathrm{~s}$ (Fig. 2e), allowing us to use a $1 \mathrm{~Hz}$ repetition rate throughout this work.

The spectrometer sensitivity is estimated by measuring the signal-to-noise ratio (SNR) of a single echo. The JPA is operated in the degenerate mode, with the phase of the pump signal chosen such that the echo signal is entirely on the amplified quadrature. With these optimal settings, the amplitude SNR of the echo shown in Fig. 3a is found to be $7 \pm 1$, one order of magnitude larger than the SNR obtained under the same conditions but with the JPA pump turned off so that it simply reflects the echo signal. This improvement is consistent with a noise reduction from $n \approx 50$ 
a

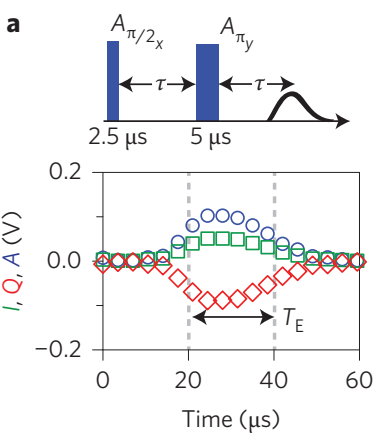

b
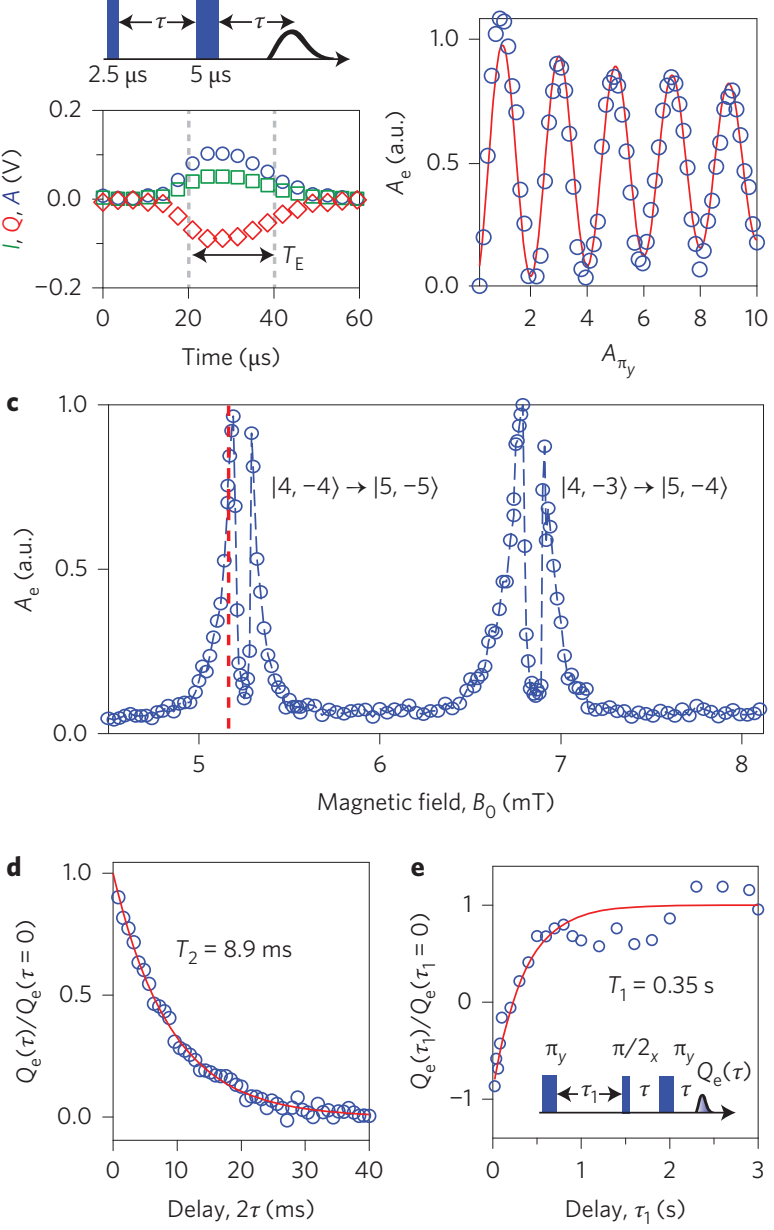

Figure 2 | Sample characterization. a, Hahn-echo sequence (top), triggering the emission of an echo (bottom). Plotted are the demodulated quadratures $I(t)$ (green squares) and $Q(t)$ (red diamonds), as well as the echo amplitude $A(t)=\sqrt{I(t)^{2}+Q(t)^{2}}$ (blue circles), from which the echo quadrature area $X_{\mathrm{e}}=\int_{-T_{E} / 2}^{+T_{E} / 2} X(t) \mathrm{d} t$ (with $X=1, Q$ ) and amplitude area $A_{\mathrm{e}}=\int_{-T_{\mathrm{E}} / 2}^{+T_{\mathrm{E}} / 2} A(t) \mathrm{d} t$ are extracted. The data are taken for $B_{0}=5.2 \mathrm{mT}$. b. Normalized amplitude echo area as a function of refocusing pulse amplitude $A_{\pi}$ (rescaled by the amplitude needed for a $\pi$ pulse) showing Rabi oscillations. Blue circles are data points and red curve is an exponentially damped cosine fit.

c, Amplitude echo area (blue circles joined by dashed lines) as a function of magnetic field $B_{0}$ showing two principal resonances, each split into a doublet due to the effect of strain on the donors below and next to the Al wire inductor. $\mathbf{d}$, As the total time $2 \tau$ between the initial $\pi / 2$ pulse and the echo is increased, the recovered $Q$ quadrature echo area decays with an exponential behaviour (red curve is a fit), yielding a spin coherence time of $T_{2}=8.9 \mathrm{~ms}$. e, The inversion recovery sequence (see inset) is used to measure the spin relaxation time $T_{1}=0.35 \mathrm{~s}$. Red curve is an exponential fit to the experimental data (blue circles).

(with the JPA off) to $n \approx 0.5$, thus close to the quantum limit, and with calibration measurements performed on the JPA itself (Supplementary Section III).

Of all the neutral Bi donors within the resonator mode volume, only those whose frequency lies within the resonator linewidth $\kappa=\omega_{0} / Q$ and that are in the $|4,-4\rangle$ state contribute to the echo signal. A rough estimate of the number of spins is therefore obtained as $N_{\mathrm{Bi}}(\kappa / \Gamma) / 9=4 \times 10^{4}$, an overestimate given that only a fraction of implanted atoms show a magnetic resonance signal due to either crystal damage or to donor ionization ${ }^{29}$. For a more accurate
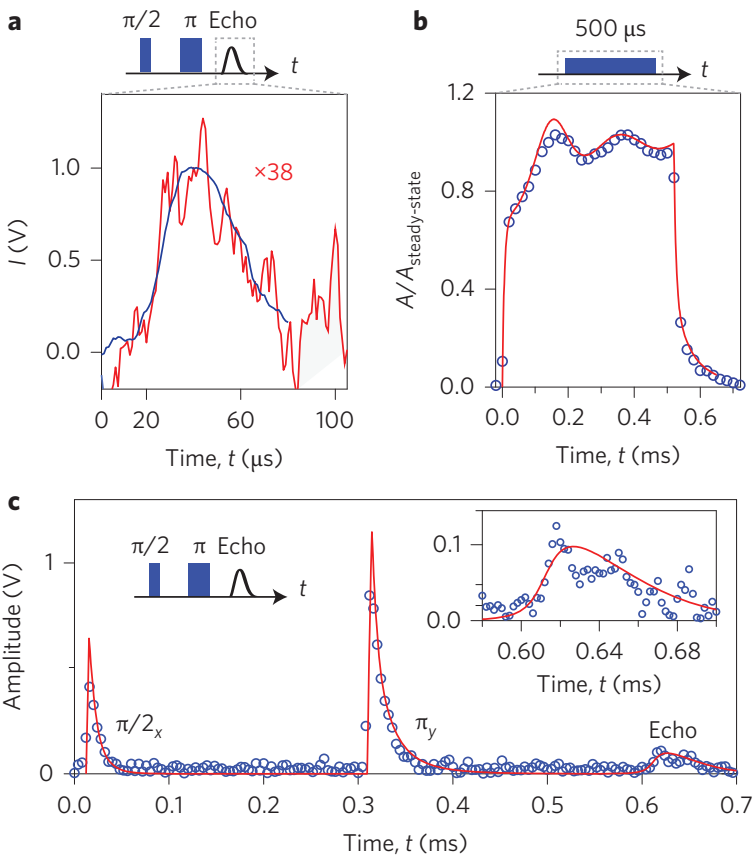

Figure 3 | Spectrometer sensitivity. a, Echo signal $I(t)$ without JPA (red curve) and with JPA in degenerate mode and $23 \mathrm{~dB}$ gain (blue curve), averaged ten times. The red curve was rescaled by the amplifier amplitude gain for comparison with the JPA-on curve. The JPA-on (JPA-off) echo has an SNR of $22 \pm 3(2 \pm 0.5)$, which translates into a single-echo SNR of $7 \pm 1$ $(0.6 \pm 0.15)$. b, Transmitted signal of a $500-\mu s-l o n g$ square drive pulse at $\omega_{0}$, averaged 1,000 times, showing temporal Rabi oscillations (circles). A simulation (curve) is used to estimate the number of spins contributing to the absorption. c, Measured microwave amplitude (circles) during an entire Hahn-echo sequence, with the JPA turned off to avoid any saturation effect. A simulation (curve) uses only the number of spins extracted from $\mathbf{b}$ and shows quantitative agreement with the measurements. These simulations indicates that the $\pi / 2$ pulse acts on $1.2 \times 10^{4}$ spins. Note that the echosignal asymmetric temporal profile (see also Fig. $2 a$ ) is due to the rapid rise and slower decay of the intra-resonator field amplitude during the control pulses because their duration is shorter than the resonator ringdown time $2 / \kappa$; this is well reproduced by the simulations.

determination, the time-dependent absorption of a microwave pulse at $\omega_{0}$ recorded and fitted to a simple model (Fig. $3 \mathrm{~b}$ and Supplementary Section IV) allows us to obtain an absolute calibration of the spin density. A whole spin-echo sequence is then measured and simulated (Fig. 3c). The quantitative agreement with the observed echo amplitude establishes (from the simulations) that $1.2 \times 10^{4}$ spins are excited during the sequence. This implies a $\sim 30 \%$ yield between number of implanted atoms and neutral donors, compatible with previous reports ${ }^{29}$.

Overall, the spectrometer can therefore detect down to $N_{\min }=1.2 \times 10^{4} / 7=1.7 \times 10^{3}$ spins with an SNR of unity in a single Hahn echo, and has a corresponding sensitivity of $1.7 \times 10^{3} \mathrm{spins} / \mathrm{Hz}^{1 / 2}$ given the $1 \mathrm{~Hz}$ repetition rate. This four orders of magnitude improvement over the state of the art is in qualitative agreement with the prediction of a simplified model (Supplementary Section IV) $N_{\min }^{(\text {th })} \simeq \sqrt{n \kappa / T_{\mathrm{E}}}(1 / g)$, where $g$ is the coupling constant of a single spin to the resonator microwave field, estimated for our geometry to be $g / 2 \pi=55 \mathrm{~Hz}$, which yields $N_{\min }^{(\text {th }}=400$ spins. The sensitivity can be further improved with a Carr-PurcellMeiboom-Gill pulse sequence, adding $m \pi_{y}$ pulses after the first echo in order to recover $m$ echoes instead of a single one, yielding an increase in SNR of $\sim m^{1 / 2}$ (ref. 7). The applicability of this technique depends on factors such as the spin coherence time $T_{2}$ of the sample and the echo duration $T_{\mathrm{E}}$. For our ${ }^{28} \mathrm{Si}: \mathrm{Bi}$ sample, 
a
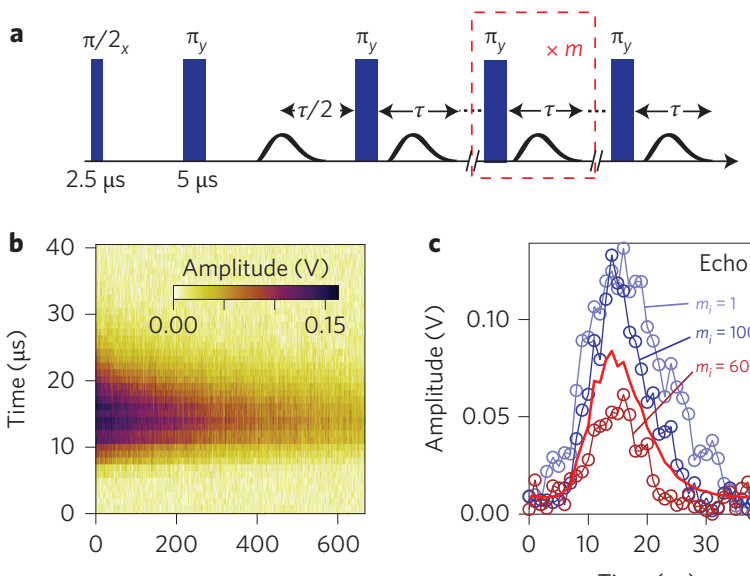

$m_{i}$

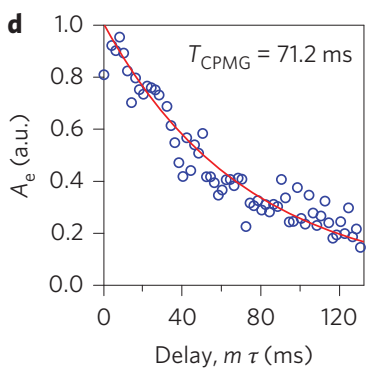

Delay, $m \tau$ (ms)
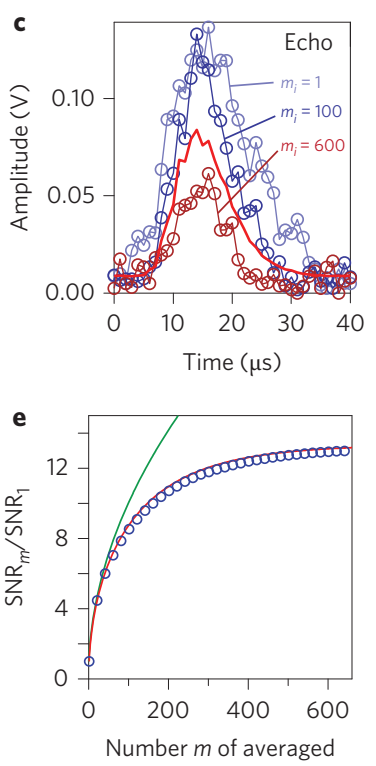

echoes

Figure 4 | Further sensitivity improvement with the Carr-PurcellMeiboom-Gill (CPMG) pulse sequence. a, A spin echo generated by any pulsed ESR experiment can be refocused by a train of $m \pi$ pulses with axes of rotation oriented along the echo phase direction, and thus used to enhance the SNR in a single shot. $\mathbf{b}$, Time dependence of the measured echo amplitude as a function of echo number $m_{i}$, over the course of a single sequence comprising $m=650 \pi$ pulses separated by $\tau=200 \mu$ s. c, Three time traces (circles) of echoes $m_{i}=1$ (light blue), $m_{i}=100$ (dark blue) and $m_{i}=600$ (dark red) are explicitly shown from $\mathbf{b}$. Solid line shows the average over all $m=650$ echoes. d, Decay of echo area $A_{\mathrm{e}}$ as a function of total delay between the initial $\pi / 2$ pulse and the echo (circles), fitted with an exponential decay (curve) of time constant $T_{\mathrm{CPMG}}=71 \mathrm{~ms}$. e, SNR improvement obtained by averaging the first $m$ echoes, as a function of the number $m$ of $\pi$ pulses within the CPMG sequence (circles), showing a tenfold improvement. In the absence of decoherence, the SNR should follow $\mathrm{m}^{1 / 2}$ (green curve). With decoherence (red curve) the SNR levels off, and eventually decays for higher $m$.

up to 600 echoes are obtained, as shown in Fig. 4, with a corresponding tenfold increase in the SNR and an unprecedented sensitivity of 150 spins in a single shot, or 150 spins/ $\mathrm{Hz}^{1 / 2}$.

A wide range of species, including molecular magnets, Gd spin-labels ${ }^{3}$ and high-spin defects in solids, can be studied by ESR at low magnetic fields using the $\mathrm{Al}$ thin-film resonator demonstrated here. Operation in larger magnetic fields $(\sim 0.3 \mathrm{~T})$ would enable the most general application of this method to other spin species and could be achieved by fabricating the microresonator from higher critical field superconductors such as $\mathrm{Nb}$ (ref. 4) or NbTiN (ref. 30). Our results thus open the way to performing ESR spectroscopy on nanoscale samples such as single cells, small molecular ensembles, nanoparticles and nanodevices. We predict that a further two orders of magnitude sensitivity enhancement is possible by reducing the resonator transverse dimensions down to the nanometre scale, which would then be sufficient for detecting individual electron spins.

\section{References}

1. Schweiger, A. \& Jeschke, G. Principles of Pulse Electron Paramagnetic Resonance (Oxford Univ. Press, 2001).

2. Yoshimura, T. et al. In vivo EPR detection and imaging of endogenous nitric oxide in lipopolysaccharide-treated mice. Nature Biotechnol. 14, 992-994 (1996).

3. Garbuio, L. et al. Orthogonal spin labeling and Gd(III)nitroxide distance measurements on bacteriophage t4-lysozyme. J. Phys. Chem. B 117, 3145-3153 (2013).

4. Sigillito, A. J. et al. Fast, low-power manipulation of spin ensembles in superconducting microresonators. Appl. Phys. Lett. 104, 222407 (2014).

5. Artzi, Y., Twig, Y. \& Blank, A. Induction-detection electron spin resonance with spin sensitivity of a few tens of spins. Appl. Phys. Lett. 106, 084104 (2015).

6. Hahn, E. L. Spin echoes. Phys. Rev. 80, 580-594 (1950).

7. Mentink-Vigier, F. et al. Increasing sensitivity of pulse EPR experiments using echo train detection schemes. J. Magn. Reson. 236, 117-125 (2013).

8. Wrachtrup, J., Von Borczyskowski, C., Bernard, J., Orritt, M. \& Brown, R. Optical detection of magnetic resonance in a single molecule. Nature 363, 244-245 (1993).

9. Grinolds, M. S. et al. Subnanometre resolution in three-dimensional magnetic resonance imaging of individual dark spins. Nature Nanotech. 9, 279-284 (2014).

10. Hoehne, F. et al. Lock-in detection for pulsed electrically detected magnetic resonance. Rev. Sci. Instrum. 83, 043907 (2012).

11. Morello, A. et al. Single-shot readout of an electron spin in silicon. Nature 467, 687-691 (2010).

12. Manassen, Y., Hamers, R. J., Demuth, J. E. \& Castellano, A. J. Jr. Direct observation of the precession of individual paramagnetic spins on oxidized silicon surfaces. Phys. Rev. Lett. 62, 2531-2534 (1989).

13. Rugar, D., Yannoni, C. S. \& Sidles, J. A. Mechanical detection of magnetic resonance. Nature 360, 563-566 (1992).

14. Wallace, W. J. \& Silsbee, R. H. Microstrip resonators for electron-spin resonance. Rev. Sci. Instrum. 62, 1754-1766 (1991).

15. Narkowicz, R., Suter, D. \& Stonies, R. Planar microresonators for EPR experiments. J. Magn. Reson. 175, 275-284 (2005).

16. Benningshof, O. W. B., Mohebbi, H. R., Taminiau, I. A. J., Miao, G. X. \& Cory, D. G. Superconducting microstrip resonator for pulsed ESR of thin films. J. Magn. Reson. 230, 84-87 (2013).

17. Bergeal, N. et al. Phase-preserving amplification near the quantum limit with a Josephson ring modulator. Nature 465, 64-68 (2010).

18. Zhou, X. et al. Highgain weakly nonlinear flux-modulated Josephson parametric amplifier using a squid array. Phys. Rev. B 89, 214517 (2014).

19. Caves, C. M. Quantum limits on noise in linear amplifiers. Phys. Rev. D 26, 1817-1839 (1982).

20. Castellanos-Beltran, M. A., Irwin, K. D., Hilton, G. C., Vale, L. R. \& Lehnert, K. W. Amplification and squeezing of quantum noise with a tunable Josephson metamaterial. Nature Phys. 4, 929-931 (2008).

21. Vijay, R., Slichter, D. H. \& Siddiqi, I. Observation of quantum jumps in a superconducting artificial atom. Phys. Rev. Lett. 106, 110502 (2011).

22. Teufel, J. D., Donner, T., Castellanos-Beltran, M. A., Harlow, J. W. \& Lehnert, K. W. Nanomechanical motion measured with an imprecision below that at the standard quantum limit. Nature Nanotech. 4, 820-823 (2009).

23. Stehlik, J. et al. Fast charge sensing of a cavity-coupled double quantum dot using a Josephson parametric amplifier. Phys. Rev. Appl. 4, 014018 (2015).

24. Hatridge, M., Vijay, R., Slichter, D. H., Clarke, J. \& Siddiqi, I. Dispersive magnetometry with a quantum limited SQUID parametric amplifier. Phys. Rev. B 83, 134501 (2011).

25. Feher, G. Electron spin resonance experiments on donors in silicon. i. Electronic structure of donors by the electron nuclear double resonance technique. Phys. Rev. 114, 1219-1244 (1959).

26. Morley, G. W. et al. The initialization and manipulation of quantum information stored in silicon by bismuth dopants. Nature Mater. 9, 725-729 (2010).

27. Dreher, L. et al. Electroelastic hyperfine tuning of phosphorus donors in silicon. Phys. Rev. Lett. 106, 037601 (2011).

28. Wolfowicz, G. et al. Atomic clock transitions in silicon-based spin qubits. Nature Nanotech. 8, 561-564 (2013).

29. Weis, C. D. et al. Electrical activation and electron spin resonance measurements of implanted bismuth in isotopically enriched silicon-28. Appl. Phys. Lett. 100, 172104 (2012)

30. Ranjan, V. et al. Probing dynamics of an electron-spin ensemble via a superconducting resonator. Phys. Rev. Lett. 110, 067004 (2013).

\section{Acknowledgements}

The authors acknowledge technical support from P. Sénat, D. Duet, J.-C. Tack, P. Pari, P. Forget, as well as useful discussions within the Quantronics Group. The authors also acknowledge support from the European Community's Seventh Framework Programme (FP7/2007-2013) through European Research Council grants nos. 615767 (CIRQUSS), 279781 (ASCENT) and 630070 (quRAM) and through the QIPC project SCALEQIT, and from C'Nano IdF through the QUANTROCRYO project. J.J.L.M. is supported by the Royal 
Society. C.C. Lo is supported by the Royal Commission for the Exhibition of 1851 . B. Julsgaard and K. Mølmer acknowledge support from the Villum Foundation. C.D.W. and T.S. acknowledge support from the Office of Science of the US Department of Energy under contract no. DE-AC02-05CH11231.

\section{Author contributions}

A.B., J.J.P., J.J.L.M. and P.B. designed the experiment. X.Z. and D.V. designed and fabricated the Josephson parametric amplifier. C.C.L., C.D.W., T.S. and M.L.W.T. provided the Biimplanted isotopically purified Si sample. A.B., J.J.P., and Y.K. fabricated the sample and performed the measurements. A.B., J.J.P., Y.K., J.J.L.M. and P.B. analysed the data. A.B., J.J.P., B.J. and K.M. performed the numerical simulations. J.J.L.M., D.E., D.V. and P.B. supervised the project. A.B., J.J.P., Y.K., M.S., D.V., D.E., B.J., K.M., J.J.L.M. and P.B. all contributed to the writing of the paper.

\section{Additional information}

Supplementary information is available in the online version of the paper. Reprints and permissions information is available online at www.nature.com/reprints. Correspondence and requests for materials should be addressed to P.B.

\section{Competing financial interests}

The authors declare no competing financial interests. 